\title{
Localization Method for a Magnetic Capsule Endoscope Propelled by a Rotating Magnetic Dipole Field
}

\author{
Katie M. Popek, Arthur W. Mahoney, and Jake J. Abbott
}

\begin{abstract}
Previous research on the localization of wireless capsule endoscopes with magnetic fields and sensors has typically utilized incremental methods. This paper provides a noniterative solution to determine the six degree-of-freedom (6DOF) position and orientation of a wireless capsule endoscope being actuated by a rotating magnetic dipole. Non-iterative solutions in the past have only been used to locate immobile objects. We experimentally demonstrate that our algorithm calculates the 6-DOF position and orientation of capsules that are truly stationary as well as those that are operated in the "step-out" regime, where the magnetic field is rotated too quickly for the capsule to rotate synchronously, but the capsule does undergo chaotic movement.
\end{abstract}

\section{INTRODUCTION}

Utilizing magnetics for actuation and localization of capsule endoscopes in the gastro-intestinal tract has become an active area of research. If the capsule is being controlled through magnetic actuation [1]-[6], little or no additional equipment is required to localize the capsule using magnetic fields. There are two main approaches for magnetic localization. The first is to use external sensors to localize a small magnet placed inside of the capsule [7]-[9]. The second method involves placing magnetic sensors inside the capsule and localizing relative to an external magnetic source [10][12]. Irrespective of where the sensors are arranged, there are numerous different methods of utilizing the collected data to determine the capsule's position and orientation. The most popular approach is to use incremental methods [11], [13]. These iterative approaches employ optimization algorithms that depend on an initial guess of the solution. Poor initial conditions can cause convergence to a local minimum instead of the true position and orientation. To overcome this, [14] and [7] combine two algorithms: the first gives a good approximation for initial conditions, which are then used in a non-linear optimization algorithm. The use of two algorithms further increases complexity and computation time. Other approaches include utilizing neural networks [10] and using precalculated data along with on-board sensing to determine the capsule's position and orientation [12], but to date these methods are somewhat time intensive.

Paperno et al. [15] demonstrated that the magnitude of a rotating magnetic field generated by a rotating magnetic dipole fluctuates elliptically at every point in space. The long

This work is supported by the National Science Foundation under grants 0952718 and 0654414.

K. M. Popek and A. W. Mahoney are with the School of Computing, and J. J. Abbott is with the Department of Mechanical Engineering, University of Utah, Salt Lake City, UT 84112, USA. email: \{katie.popek, jake.abbott\}@utah.edu

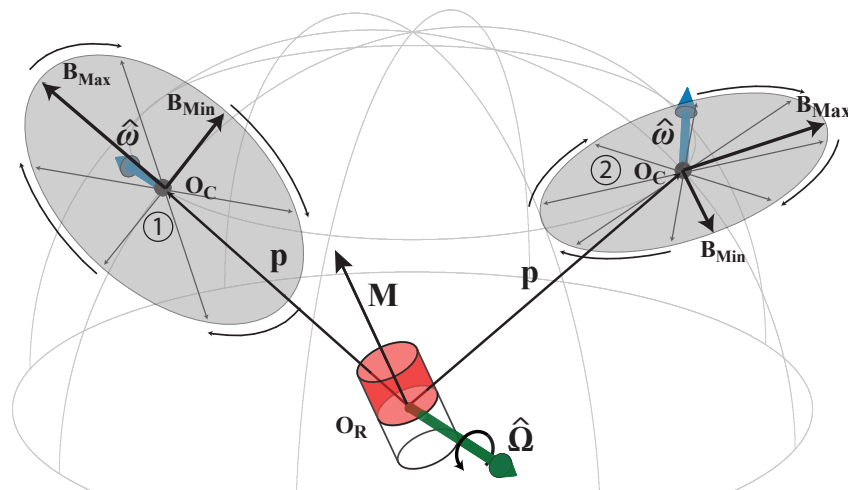

Fig. 1. The magnetic field magnitude generated by a rotating magnetic dipole, such as a rotating permanent magnet (RPM), fluctuates elliptically at any point in space. The size of the ellipse changes with the distance between the RPM and the point.

axis of the ellipse is defined by the maximum-magnitude magnetic field $\mathbf{B}_{\max }$, and the short axis is defined by the minimum-magnitude magnetic field $\mathbf{B}_{\min }$, as shown in Fig. 1. With this knowledge they were able to solve for the position and orientation of a static magnetic sensor in a known quadrant, using equations derived from the point-dipole model. Their result is based on finding the angles of a rotating electromagnetic coil using a phase-lock technique. We have reworked this solution in a linear-algebraic approach, which we believe is more straightforward. In addition, there are known errors in the orientation algorithm of [15], which have been corrected here.

Inspired by [15], Ge et al. [16] use two orthogonal coils in a "searching" technique to determine the position of the coils that produces the maximum field at the sensor. The accuracy of this method is directly dependent on how well it is able to align the coils with the maximum measurements. This also restricts the motion of the manipulator and makes it challenging to actuate the capsule with the same coil system being used for localization.

In our lab we are investigating the use of a single rotating permanent magnet (RPM) positioned in space by a 6-degreeof-freedom (6-DOF) robotic manipulator to actuate wireless capsule endoscopes from arbitrary positions [5]. To most efficiently control the capsule, its state in the field (e.g., Is the capsule rotating synchronously with the field or has it stepped out of synchronization?) must be known so appropriate adjustments to the RPM's pose and speed can 


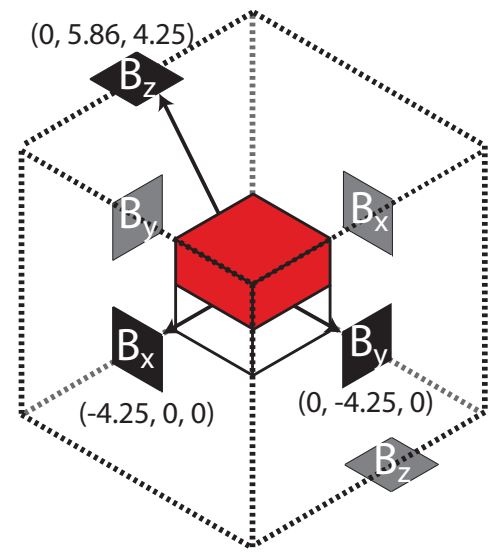

Fig. 2. The sensor configuration from [17] with each sensor labeled with the field direction it is measuring and its position from the center of the magnet in millimeters. The grey sensors are not visible from this angle, but are located at the equivalent negative position.

be made to actuate the capsule in the desired fashion. We have previously created a three-axis magnetic sensor array using six linear Hall-effect sensors that was designed for the purpose of determining the state of the capsule [17]. This sensor configuration, shown in Fig. 2, was chosen because the internal magnet's field is parallel to each sensor at its position, such that the sensor measurements are not corrupted by the close proximity of the internal magnet. In addition, it is possible to average the two sensor readings in each cardinal direction to approximate the three-dimensional magnetic field vector at the center of the capsule's internal magnet with negligible interference from the magnet itself. Using this previously designed sensor arrangement, our goal is to localize the capsule's position and orientation relative to the RPM, and thus relative to the world coordinate frame, as the RPM is used to actuate the capsule.

In our proposed actuation/localization concept, the RPM is used to actuate the capsule at relatively slow synchronized frequencies, and periodically the RPM quickly completes an integer number of rotations above the step-out frequency of the capsule to update the localization estimate (step-out occurs when the field is rotating too quickly, given the magnitude of the field, for the capsule to remain synchronized with the field), after which it returns to the synchronous actuation mode. We have already demonstrated that this synchronous actuation mode is quite robust against localization errors [18]. In this paper, we propose a localization method that provides a fast alternative to iterative algorithms, and is straightforward to implement. Unlike previous non-iterative approaches, which focused exclusively on stationary objects, this solution permits localizing both when the capsule is stationary and also when it is rotating chaotically above the step-out frequency.

\section{Position Detection}

Because the orientation of the sensor arrangement is not known a priori, we determine the position based strictly on the magnitude of the field being measured by the sensors, and the pose of the RPM dipole moment when the maximum and minimum magnitudes occur. Using this method, it is possible to reduce the potential capsule positions to four points. However, with our understanding of where the RPM is with respect to the patient, narrowing down the capsule's position between these four points in space may be possible without any further rotations of the RPM. Regardless, changing the axis of rotation for the RPM and performing a second rotation would reduce the possible capsule locations to a single point, and we have already demonstrated the ability to change the RPM position in this fashion while still continuously actuating the capsule [5].

Assume an RPM is positioned in space by a 6-DOF robotic manipulator. The center of the RPM is the origin for the robot's end-effector coordinate frame, $\mathbf{O}_{R}$. Any vector in this coordinate frame will be denoted throughout this paper with an " $R$." The center of the small permanent magnet placed inside the capsule is the origin of the capsule's coordinate frame, $\mathbf{O}_{C}$. Any vector in this coordinate frame will be represented with a " $C$." All unit vectors are denoted with a "^". As depicted in Fig. 1, there exists some vector $\mathbf{p}$ that describes the shift in position between $\mathbf{O}_{R}$ and $\mathbf{O}_{C}$. In addition, there exists some rotation matrix $\mathbb{R}_{R C}$ that rotates the capsule's coordinate frame to align it with the robot's. By solving for the position vector $\mathbf{p}$ and the rotation matrix $\mathbb{R}_{R C}$, the capsule's relative position and orientation to the external magnetic source is known.

Four assumptions were made to develop this method: (1) The direction of the magnetic dipole moment $\mathbf{M}$ for the RPM is known as it rotates. In practice, this is measured with an optical encoder in our current experimental setup. (2) The RPM rotates about $\hat{\Omega}$, so that $\mathbf{M}$ rotates in the plane normal to $\hat{\Omega}$. (3) $\mathbf{B}_{\min }$ and $\mathbf{B}_{\max }$ are accurately known using sensors placed inside of the capsule. (4) The point-dipole model accurately represents the magnetic field generated by the RPM (errors induced with this assumption for a nonspherical RPM are quantified in [19]). With this final assumption, the field at any capsule position $\mathbf{p}$ is given by [20]:

$$
\mathbf{B}(\mathbf{p})=\frac{\mu_{0}}{4 \pi|\mathbf{p}|^{3}}\left(3 \hat{\mathbf{p}} \hat{\mathbf{p}}^{T}-\mathbb{I}\right) \mathbf{M}=\frac{\mu_{0}}{4 \pi|\mathbf{p}|^{3}} \mathbb{B} \mathbf{M}
$$

where $\mu_{0}$ is the permeability of free space, $\mathbb{I}$ is the identity matrix, and $\mathbb{B}$ is a symmetric matrix that depends only on the direction of $\hat{\mathbf{p}}$.

As described in [5], at any position in space $\mathbf{p}$, the magnitude of the field $|\mathbf{B}|$ fluctuates elliptically:

$$
|\mathbf{B}|=\frac{\mu_{0}|\mathbf{M}|}{4 \pi|\mathbf{p}|^{3}} \sqrt{1+3\left(\hat{\mathbf{M}}^{T} \hat{\mathbf{p}}\right)^{2}}
$$

$\mathbf{B}$ is always normal to the local rotation axis $\hat{\boldsymbol{\omega}}$, as shown in Fig. 1. The maximum and minimum field magnitudes

$$
\begin{gathered}
\left|\mathbf{B}_{\max }\right|=\frac{\mu_{0}|\mathbf{M}|}{4 \pi|\mathbf{p}|^{3}} \sqrt{1+3|\tilde{\mathbf{p}}|^{2}} \\
\left|\mathbf{B}_{\min }\right|=\frac{\mu_{0}|\mathbf{M}|}{4 \pi|\mathbf{p}|^{3}}
\end{gathered}
$$


occur when the RPM's dipole moment $\mathbf{M}$ is parallel and perpendicular to $\tilde{\mathbf{p}}$, respectively, where $\tilde{\mathbf{p}}$ is the projection of $\hat{\mathbf{p}}$ onto the plane normal to $\hat{\mathbf{\Omega}}$ (i.e., the plane in which $\mathbf{M}$ resides).

The magnitude of the position vector, which is the distance from the center of the capsule's magnet to the center of the RPM, can be computed by rearranging (4):

$$
|\mathbf{p}|=\left(\frac{\mu_{0}|\mathbf{M}|}{4 \pi\left|\mathbf{B}_{\min }\right|}\right)^{\frac{1}{3}}
$$

This gives a spherical surface of possible capsule locations with a radius of $|\mathbf{p}|$ from the center of the RPM.

To find the direction $\hat{\mathbf{p}}$, the angle $\theta$ between $\hat{\mathbf{M}}$ and $\hat{\mathbf{p}}$ is calculated by rearranging (2):

$$
\cos ^{2} \theta=\left(\hat{\mathbf{M}}^{T} \hat{\mathbf{p}}\right)^{2}=\frac{1}{3}\left(\left(\frac{4 \pi|\mathbf{B}||\mathbf{p}|^{3}}{\mu_{0}|\mathbf{M}|}\right)^{2}-1\right)
$$

There are four possible solutions for $\theta$. For numerical stability, we use arctan instead of arccos to solve for $\theta$ :

$$
\theta=\arctan \left( \pm \sqrt{\frac{\sin ^{2} \theta}{\cos ^{2} \theta}}\right)=\left( \pm \sqrt{\frac{1-\cos ^{2} \theta}{\cos ^{2} \theta}}\right)
$$

As described in [5], when the magnitude of the magnetic field is maximized, $\mathbf{M}$ is parallel to $\tilde{\mathbf{p}}$. The angle solved for in (7) is thus also the angle between $\hat{\mathbf{p}}$ and $\tilde{\mathbf{p}}$.

Assuming that $\hat{\boldsymbol{\omega}}$ is the rotation axis of the magnetic field at position $\mathbf{p}, \hat{\boldsymbol{\omega}}$ will always be perpendicular to the plane in which $\mathbf{B}$ resides, resulting in the following equation:

$$
\mathbf{B}^{T} \hat{\boldsymbol{\omega}}=\frac{\mu_{0}}{4 \pi|\mathbf{p}|^{3}} \mathbf{M}^{T} \mathbb{B} \hat{\boldsymbol{\omega}}=0
$$

The axis of rotation for the RPM $\hat{\Omega}$ can then be uniquely solved for because $\mathbb{B} \hat{\boldsymbol{\omega}}$ is constant for a given $\mathbf{p}$ and $\mathbf{M}^{T} \hat{\boldsymbol{\Omega}}=$ 0 for all $\mathbf{M}$. Therefore, only one solution for $\hat{\Omega}$ exists that satisfies (8) and remains constant regardless of $\mathbf{M}$ rotation:

$$
\hat{\Omega}=\widehat{\mathbb{B} \hat{\omega}}
$$

By expanding (9), it is possible to prove that the rotation axis $\hat{\Omega}$, the capsule's position $\mathbf{p}$, and the rotation axis of the local magnetic field $\hat{\boldsymbol{\omega}}$ are coplanar as follows. Let $c$ represent a scalar constant for normalization, and since from [5], $\mathbb{B}^{-1}=\frac{1}{2}(\mathbb{B}-\mathbb{I}),(9)$ can be written as

$$
\hat{\boldsymbol{\omega}}=c \mathbb{B}^{-1} \hat{\boldsymbol{\Omega}}=\left(\frac{3 c\left(\hat{\mathbf{p}}^{T} \hat{\mathbf{\Omega}}\right)}{2}\right) \hat{\mathbf{p}}-c \hat{\mathbf{\Omega}}
$$

and thus $\hat{\boldsymbol{\omega}}$ is in the span of $\hat{\mathbf{p}}$ and $\hat{\boldsymbol{\Omega}}$, and therefore $\hat{\boldsymbol{\omega}}, \hat{\mathbf{p}}$, and $\hat{\Omega}$ are coplanar.

Using Rodrigues' formula it is possible to rotate a vector $\mathbf{v}$ about an arbitrary axis $\hat{\mathbf{k}}$ by some angle $\theta[21]$ :

$$
\mathbf{v}^{\prime}=\mathbf{v} \cos \theta+(\hat{\mathbf{k}} \times \mathbf{v}) \sin \theta+\hat{\mathbf{k}}(\hat{\mathbf{k}} \cdot \mathbf{v})(1-\cos \theta)
$$

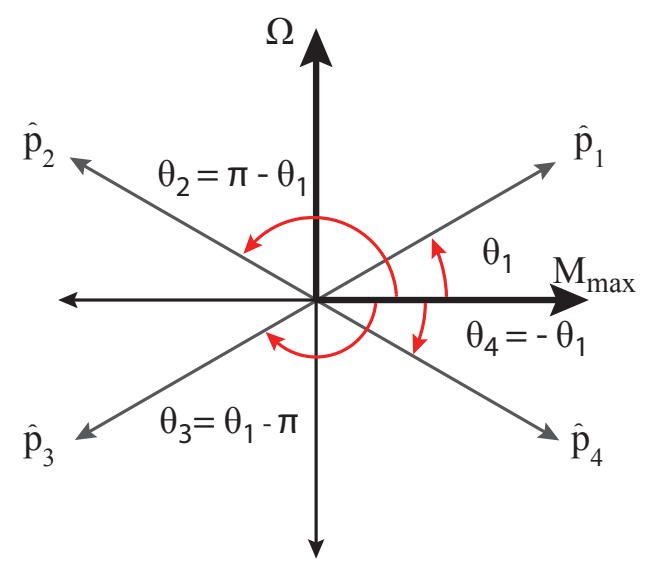

Fig. 3. The four possible $\hat{\mathbf{p}}$ are shown as they would appear in the $\mathbf{\Omega}-\mathbf{M}_{\max }$ plane. These result from the four solutions to $\theta$ from (6).

Because $\hat{\mathbf{p}}$ and $\hat{\boldsymbol{\Omega}}$ are coplanar and $\tilde{\mathbf{p}}$ is parallel to $\hat{\mathbf{M}}_{\max }$, by choosing $\hat{\mathbf{k}}=\hat{\mathbf{M}}_{\max } \times \hat{\mathbf{\Omega}}=\hat{\mathbf{M}}_{\min }$, where $\hat{\mathbf{M}}_{\max }$ and $\hat{\mathbf{M}}_{\min }$ are the directions of $\mathbf{M}$ at the maximum and minimum field magnitudes experienced by the capsule, $\hat{\mathbf{k}}$ is normal to $\hat{\mathbf{p}}$ and $\tilde{\mathbf{p}}$. Using (11), we can rotate $\tilde{\mathbf{p}}$, or equivalently $\hat{\mathbf{M}}_{\max }$, by $\theta$ about $\hat{\mathbf{k}}$ to calculate $\hat{\mathbf{p}}$. There are four possibilities because of the four possible angles; these are shown in Fig. 3. Finally, the position vector between the RPM and the capsule can be solved for by $\mathbf{p}=|\mathbf{p}| \hat{\mathbf{p}}$. Pseudocode to implement the position localization method is given as Algorithm 1 .

\section{ORIENTATION DEteCtion}

As depicted in Fig. 1, when the field rotates around $\hat{\omega}$, it produces an ellipse at any point in space. The size is dependent on the distance to the point. As long as the distance remains the same, the size of the ellipse will remain constant. If the capsule is rotated, the two coordinate frames are no longer aligned and the ellipse in the capsule's frame of reference will be rotated from the original ellipse. The rotation transformation between the capsule's and the robot's frame of reference will be the same as the rotation between the two ellipses.

Because the position was previously found, we can determine the magnetic field at the capsule's position using (1). This corresponds to the measurements that we could expect if the capsule's frame of reference was aligned with the robot's. The maximum and minimum field measurements were previously measured in the capsule's frame of reference to calculate the position. Taking the cross product of these two perpendicular vectors $\mathbf{B}_{C \text { min }}$ and $\mathbf{B}_{C \text { max }}$ results in a third vector $\mathbf{B}_{C \perp}=\mathbf{B}_{C \max } \times \mathbf{B}_{C \text { min }}$ that is normal to the previous two. Creating a $3 \times 3$ matrix $\mathbb{A}_{C}$ with these three vectors as columns, and the equivalent $\mathbb{A}_{R}$ calculated using the point-dipole model (1), it is possible to calculate the rotation matrix between the two coordinate systems:

$$
\mathbb{R}_{R C}=\mathbb{A}_{R} \mathbb{A}_{C}^{-1}
$$




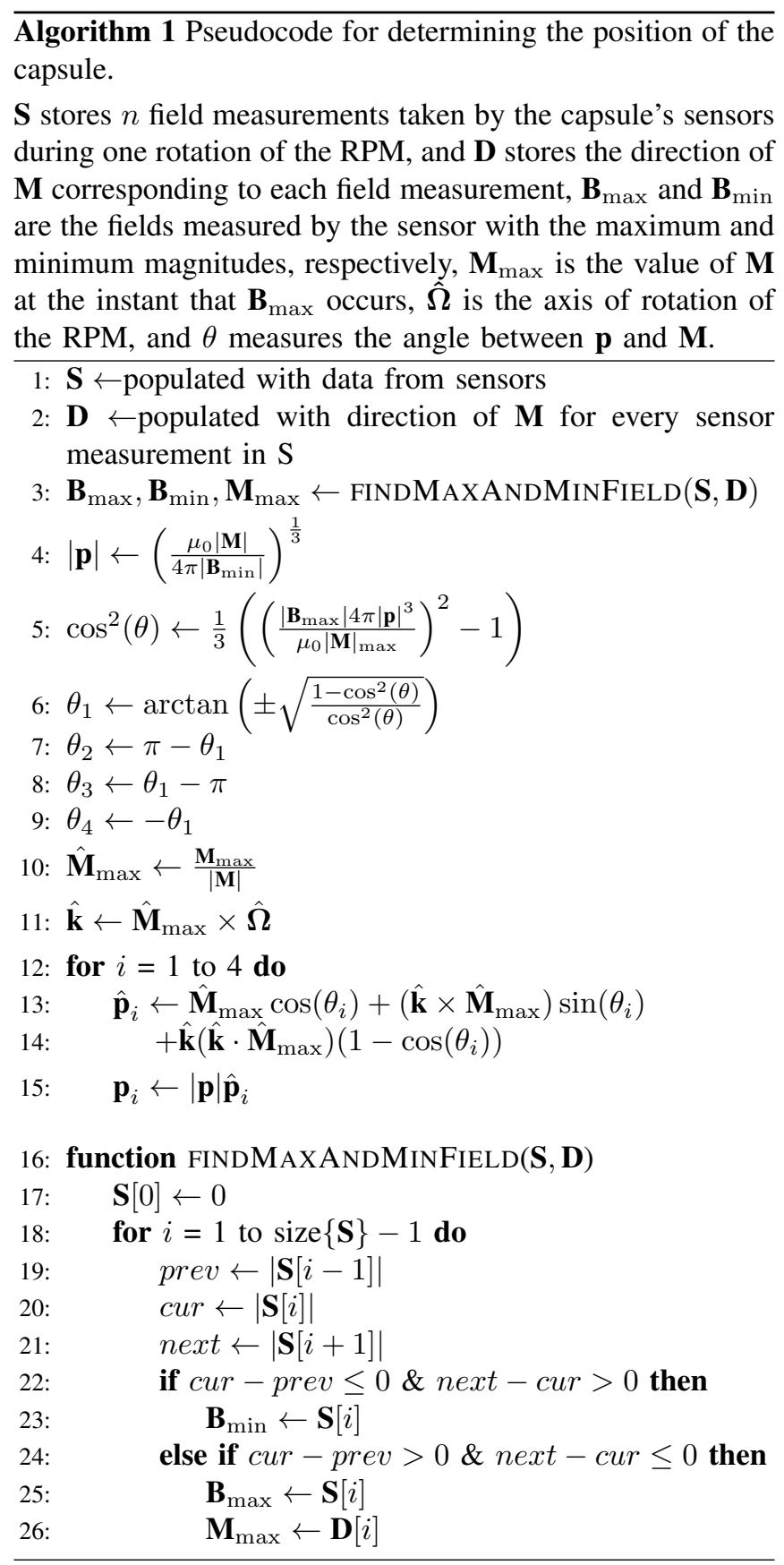

where

$$
\begin{aligned}
\mathbb{A}_{C} & =\left[\begin{array}{lll}
\mathbf{B}_{C \max } & \mathbf{B}_{C \min } & \mathbf{B}_{C \perp}
\end{array}\right] \\
\mathbb{A}_{R} & =\left[\begin{array}{lll}
\mathbf{B}_{R \max } & \mathbf{B}_{R \min } & \mathbf{B}_{R \perp}
\end{array}\right]
\end{aligned}
$$

Although this method will work accurately to calculate the orientation when the capsule is completely stationary and the sensors are free of noise, in practice three data points are not sufficient. To receive a better estimate, we can use every sensor measurement gathered during one complete rotation of the RPM (or at least more than three). By adding every measurement taken as a column to $\mathbb{A}_{C}$ and the corresponding expected measurement to $\mathbb{A}_{R}$, each becomes a $3 \times n$ matrix where $n$ is the number of measurements taken in one rotation of the RPM. (12) then uses a pseudo-inverse to solve for the rotation matrix using a least-squares approach. Assuming that the capsule is in step-out and not able to rotate synchronously with the field, it will oscillate chaotically trying to align itself with the external magnetic field. The motion will average to an orientation that can be found using this method.

When the capsule is in the step-out regime, there is no longer a pure rotation matrix that aligns the robot and capsule coordinate frames because the capsule has moved throughout the data set. Instead, we must find the rotation matrix that best aligns the frames. From [22], it is known that a rotation matrix can be written in exponential form:

$$
\mathbb{R}=e^{\mathbb{S}}
$$

where $\mathbb{S}$ is a skew-symmetric matrix:

$$
\mathbb{S}=\left[\begin{array}{ccc}
0 & -s_{3} & s_{2} \\
s_{3} & 0 & -s_{1} \\
-s_{2} & s_{1} & 0
\end{array}\right]
$$

with the rotation axis $\hat{\mathbf{k}}$ and the angle $\theta$ embedded in it:

$$
\hat{\mathbf{k}} \theta=\left[\begin{array}{l}
s_{1} \\
s_{2} \\
s_{3}
\end{array}\right]
$$

Using the rotation matrix that was calculated by (12), it is possible to solve for the skew-symmetric matrix $\mathbb{S}=$ $\ln (\mathbb{R})$. Because of the noisy data, $\mathbb{S}$ will not necessarily be skew-symmetric. By converting $\mathbb{S}$ to a true skew-symmetric matrix - setting the small diagonal terms to zero, and averaging the appropriate values to estimate $s_{1}, s_{2}$, and $s_{3}$-a best-fit rotation matrix can be found by substituting this new true skew-symmetric matrix into (13).

\section{EXPERIMENTAL SETUP}

Our method was tested by localizing a prototype capsule that measures $25 \mathrm{~mm}$ in diameter by $50.5 \mathrm{~mm}$ in length. The capsule rotates in a clear acrylic tube that was rigidly held in place. The RPM was chosen as a $25.4 \mathrm{~mm}$ diameter by $25.4 \mathrm{~mm}$ long cylindrical Grade-N42 NdFeB permanent magnet and was attached to the end-effector of a Yaskawa Motoman MH5 6-DOF robotic manipulator. The RPM was rotated with a Maxon $24 \mathrm{~V}$ A-Max DC motor in conjunction with an Advanced Motion Controls servo control drive and amplifier. This setup is shown in Fig. 4.

The prototype capsule measures the magnetic field at the center of its $108 \mathrm{~mm}^{3}$ Grade-N52 cubic internal permanent magnet using six one-axis Allegro A1392 linear Hall-effect sensors with a sensitivity of $25 \mathrm{~V} / \mathrm{T}$ and a range of $\pm 64 \mathrm{mT}$. The capsule is pictured in Fig. 5. The sensor measurements are taken at $200 \mathrm{~Hz}$ and are wirelessly transmitted from the capsule to a computer where the localization algorithm is run. The sensors were calibrated before every test to remove any offset from the capsule's internal magnet; the Earth's magnetic field (approximately $50 \mu \mathrm{T}$ ) is negligible.

The capsule was placed at a known position and orientation relative to the 6-DOF robot with $\mathrm{a} \pm 2 \mathrm{~mm}$ and 


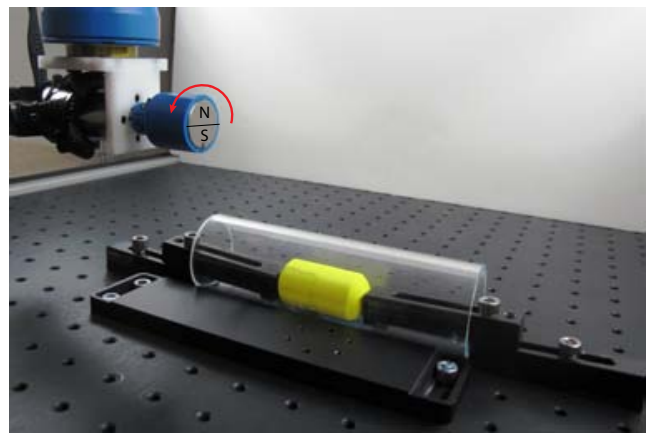

Fig. 4. Experimental setup of system with a diametrically magnetized RPM mounted to a 6-DOF robot. The capsule was held stationary (shown) and allowed to rotate in step-out (not shown) in the clear acrylic tube fixed rigidly in place.

\pm 5 degree accuracy, respectively. This relative distance was held constant throughout each test. When the capsule was being tested in step-out it was able to oscillate chaotically; otherwise it was held rigid. The RPM was rotated for 30 seconds and the field measurements collected by the sensors were wirelessly transferred back to the computer; this amount of data was collected for purposes of analysis, but in practice as little as one complete cycle is needed. Concurrently, the position of the RPM dipole was measured using an optical encoder attached to the motor that spins the RPM.

\section{Results And Discussion}

In the first test, represented in Fig. 6(a), the RPM was rotated at $5 \mathrm{~Hz}$, such that the capsule was operating in the step-out regime. It oscillated back and forth in the field trying to align its magnetic dipole moment with the RPM's. Using our developed algorithm, the position and orientation of the capsule in a known quadrant were found with total errors of $11 \mathrm{~mm}$ in the position and 11 degrees in the orientation. In Fig. 6, the yellow capsule represents the known position with respect to the external RPM, and the red capsule is the position estimated using our algorithm. In the bottom portion, the black dots are the sensor's field measurements, which have been rotated from the capsule's frame to the robot's using our calculated $\mathbb{R}_{R C}$. These measurements are overlaid on the expected magnetic field (red line) calculated using (1) at the previously estimated position.

To verify the algorithm's performance on stationary objects, a second test was conducted with the capsule held rigidly, as depicted in Fig. 6(b). The total position error decreased to $3.8 \mathrm{~mm}$, but the orientation error was slightly higher at 13 degrees; although this increase in orientation error from the step-out case is counter-intuitive, it is within the accuracy of the baseline.

These two examples are representative of the localization results that we have found experimentally with the proposed method when post-processing 30 seconds of data. With an online localization system, utilizing less data will be desirable; recall that the RPM will be briefly rotated above the step-out frequency to perform localization. Although the

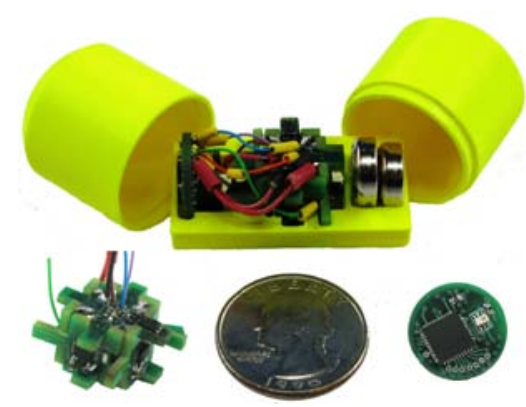

Fig. 5. From left to right, the contents of the capsule include the electronics for wireless communication, the sensor array with six Hall-effect sensors surrounding a permanent magnet, and coin-cell batteries. For a closer view the sensor array is pictured in the bottom left and the communication PCB in the lower right.

method can work with as little as one rotation of the RPM, the average total position error in the step-out case doubled when used in this manner. Using 8 rotations, the error decreases to $16 \mathrm{~mm}$, and 20 rotations results in $12.6 \mathrm{~mm}$ total error, which is only slightly higher than using 30 seconds of data (150 rotations). These errors are sufficiently small for our previously developed magnetic-actuation algorithms to be used [18]. However, further exhaustive testing of the workspace (both theoretical and experimental) still needs to be completed to fully grasp how the pose of the RPM with respect to the capsule influences the localization errors. Additionally, a Kalman filter that utilizes a model of the capsule's kinematics could significantly improve the localization.

This method was developed assuming the RPM, which rotates normal to its dipole moment, could be modeled with the point-dipole equation. In addition, it was assumed that $\mathbf{B}_{\max }, \mathbf{B}_{\min }$, and the dipole orientation could be perfectly measured. Although our method would provide perfect localization if these assumptions are true, in practical situations, this is never the case. In our hardware setup, there was a time lag between the measurements of the sensors and the dipole orientation. This led to position errors because the dipole moment pose is used in the calculation of the direction of p. We solved this by post-processing the data; however with improved synchronization, this method could be used for online localization. This will be the goal of future work.

We have yet to explore the movement of the RPM with respect to the capsule in order to improve the localization results (similar to the concept of persistent excitation), which will also eliminate the false potential positions that occur, such that the "known quadrant" assumption can be relaxed. In addition, increased accuracy may be achieved by using the developed algorithms in conjunction with Bayesian filters, such that RPM movements are chosen that best reduce uncertainty in the localization estimate.

\section{CONCLUSION}

In this paper, we developed a method to determine the position and orientation of a magnetic capsule endoscope using the same rotating dipole field that is being used to actuate/propel the capsule. The method was experimentally 
(a)

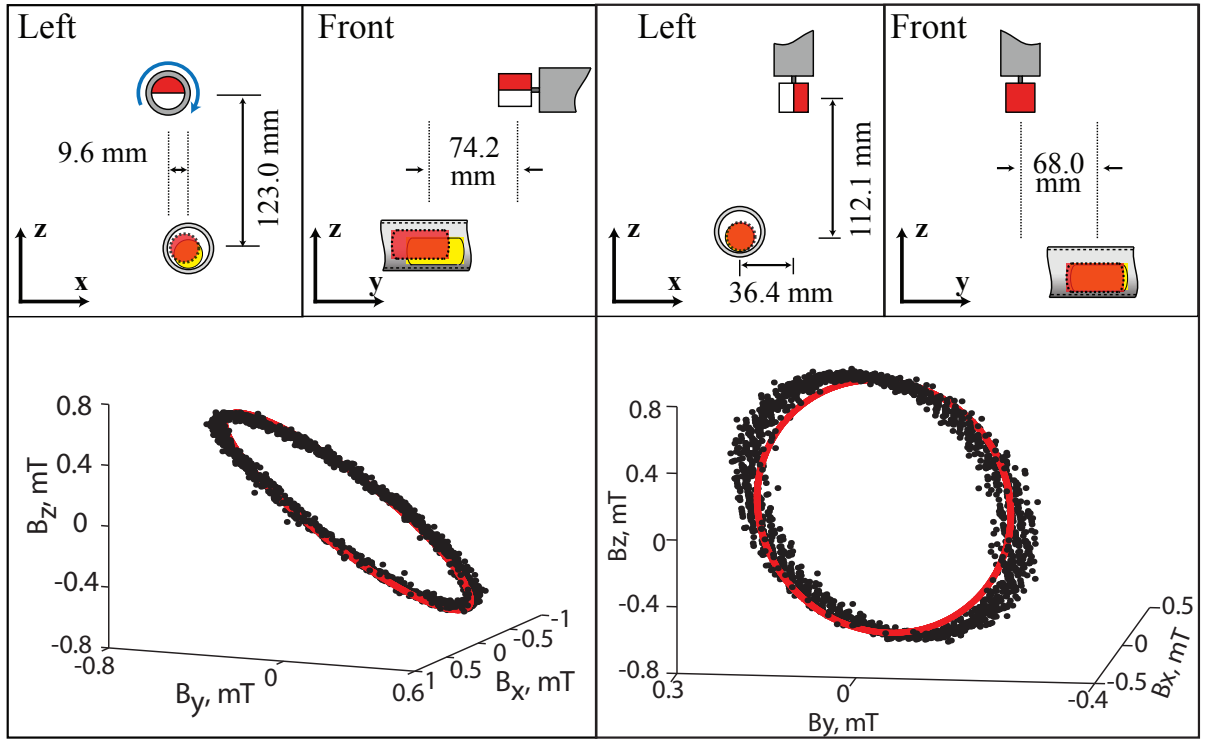

Fig. 6. The position of the capsule estimated by the localization algorithm is shown by the red capsule relative to the known position (yellow) and the RPM on the end of the robot. In (a) the RPM was rotated quickly such that the capsule was in the step-out regime, and in (b) the capsule was held rigidly. The sensor's field measurements (black dots) were rotated from the capsule's frame to the robot's and were overlaid on the expected measurements (red line) to show the measured and computed orientation beneath the respective robot position.

verified and was found to result in sufficiently small errors to be used by existing magnetic-actuation methods. In the future, this algorithm can be implemented for real-time localization of a capsule endoscope, subject to improvements in data-transfer rates.

\section{ACKNOWLEDGMENT}

The authors would like to thank Dr. Thomas Schmid for assistance with the design of the capsule prototype.

\section{REFERENCES}

[1] A. Uehara and K. Hoshina. Capsule endoscope NORIKA system. Min Invas Ther and Allied Technol, 1(1):227-334, 2003.

[2] X. Wang and M.Q.-H. Meng. Computational aspects in actuation and guidance mechanism for wireless active capsule endoscope. In IEEE Int. Conf. Intelligent Robots and Systems, pages 1198-1203, 2008.

[3] G. Ciuti, P. Valdastri, A. Menciassi, and P. Dario. Robotic magnetic steering and locomotion of capsule endoscope for diagnostic and surgical endoluminal procedures. Robotica, 28:199-207, 2010.

[4] F. Carpi, N. Kastelein, M. Talcott, and C. Pappone. Magnetically controllable gastrointestinal steering of video capsules. IEEE Trans. Biomed. Eng., 58(2):231-234, 2011.

[5] A. W. Mahoney, D. L. Cowan, K. M. Miller, and J. J. Abbott. Control of untethered magnetically actuated tools using a rotating permanent magnet in any position. In IEEE Int. Conf. Robot. Auto., pages 231234, 2012.

[6] J. Kim, Y. Kwon, and Y. Hong. Automated alignment of rotating magnetic field for inducing a continuous spiral motion on a capsule endoscope with a twistable thread mechanism. Int. J. of Prec. Eng. \& Мапи., 13(3):371-377, 2012.

[7] C. Hu, M. Li, S. Song, W. Yang, R. Zhang, and M.Q.-H. Meng. A cubic 3-axis magnetic sensor array for wirelessly tracking magnet position and orientation. IEEE Sensors J, 10(5):903-913, 2010.

[8] W. Yang, C. Hu, M. Li, M.Q.-H. Meng, and S. Song. A new tracking system for three magnetic objectives. IEEE Trans Magnetics, 46(12):4023 -4029, 2010.

[9] W. Weitschies, H. Blume, and H. Monnikes. Magnetic marker monitoring: High resolution real-time tracking of oral solid dosage forms in the gastrointestinal tract. European J Pharmaceutics and Biopharmaceutics, 74(1):93 - 101, 2010.
[10] X. Guo, G. Yan, and W. He. A novel method of three-dimensional localization based on a neural network algorithm. J of Med Eng \& Tech, 33(3):192-198, 2009.

[11] M. Kim, Y. Hong, and E. Lim. Position and orientation detection of capsule endoscopes in spiral motion. Int. J of Prec. Eng. and Manu., 11(1):31-37, 2010.

[12] M. Salerno, G. Ciuti, G. Lucarini, R. Rizzo, P. Valdastri, A. Menciassi, A. Landi, and P. Dario. A discrete-time localization method for capsule endoscopy based on on-board magnetic sensing. Meas. Sci. Technol., 23(1):015701, 2012.

[13] H. Son and K. Lee. Distributed multipole models for design and control of PM actuators and sensors. IEEE/ASME Trans. Mech., 13(2):228-238, 2008.

[14] W. Yang, C. Hu, M.Q.-H. Meng, S. Song, and H. Dai. A sixdimensional magnetic localization algorithm for a rectangular magnet objective based on a particle swarm optimizer. IEEE Trans. Mag, 45(8):3092-3099, 2009.

[15] E. Paperno, I. Sasada, and E. Leonovich. A new method for magnetic position and orientation tracking. IEEE Trans. Magn., 37(4):19381940, 2008.

[16] X. Ge, Y. Wang, N. Ding, X. Wu, Y. Wang, and Z. Fang. An electromagnetic tracking method using rotating orthogonal coils. IEEE Trans Magnetics, DOI:10.1109/TMAG.2012.2203917, 2012.

[17] K. M. Miller, A. W. Mahoney, T. Schmid, and J. J. Abbott. Proprioceptive magnetic-field sensing for closed-loop control of magnetic capsule endoscopes. In IEEE Int. Conf. Intel. Robots \& Sys., pages 1994-1999, 2012.

[18] A. W. Mahoney and J. J. Abbott. Control of untethered magnetically actuated tools with localization uncertainty using a rotating permanent magnet. In IEEE Int. Conf. Biomedical Robotics and Biomechatronics, pages 1632-1637, 2012.

[19] A. J. Petruska and J. J. Abbott. Optimal permanent-magnet geometries for dipole field approximation. IEEE Trans. Mag, 49(2):811-819, 2013.

[20] E. P. Furlani. Permanent magnet and electromechanical devices: materials, analysis, and applications. Academic Press, San Diego, California, 1st edition, 2001.

[21] D. Koks. Explorations in Mathematical Physics. Springer, New York, 1st edition, 2006.

[22] R. M. Murray, Z. Li, and S. S. Sastry. A Mathematical Introduction to Robotic Manipulation. CRC Press, Boca Raton, 1994. 